ROKSANA JURCZAK ${ }^{1}$, JULITA REGUŁA ${ }^{2}$

${ }^{1}$ Department of Food Industry Processes and Facilities

Koszalin University of Technology

${ }^{2}$ Department of Human Nutrition and Hygiene

Poznań University of Life Sciences

\title{
PLANT MATERIALS USED IN SUPPORTING THE TREATMENT OF GOUT
}

\author{
SUROWCE ROŚLINNE WYKORZYSTYWANE WE WSPOMAGANIU LECZENIA \\ DNY MOCZANOWEJ
}

\begin{abstract}
Summary
Gout (arthritis), is a disease belonging to the group of rheumatoid disorders. As a result of some disorders in the uric acid metabolism, deposition of its crystals takes place in tissues and organs. It causes pain of joints and forming of tophi and tophi in kidney. The limit of uric acid in the blood is $6.5 \mathrm{mg} \%$. Worldwide, especially in well-developed countries, the frequency of gout incidence is still increasing - more in men than women. The incidence among women is increasing only in the postmenopausal period. Methods of gout treatment are based on a comprehensive approach to patients and include primarily lifestyle changes (introduction of special diets and increased physical activity) and introduction of drugs that act as analgesic and anti-inflammatory, as well as are able to remove an excess of uric acid. For ages, in gout treatment also plant raw materials have been applied. They show a potency comparable to medicines, but usually exhibit weaker side effects. Medicine pays special attention, while curing gout, to such plants as: Aegopodium podagraria L., Harpagophytum procumbens (Devil's Claw), willow bark, birch leaves, mud meadowsweet flower, sour cherries, but also common goldenrod, nettle, horsetail and wild rose.
\end{abstract}

Key words: arthritis, diet, plants, purine, bioactive substances

\section{Introduction}

Gout (arthritis), is a disease belonging to the group of rheumatoid disorders (Lamer-Zarawska et al., 2012). It was described already in the antiquity; the first mentions 
Jurczak, R., Reguła, J. (2016). Plant materials used in supporting the treatment of gout. Nauka Przyr. Technol., 10, 4, \#58. DOI: http://dx.doi.org/10.17306/J.NPT.2016.4.58

come from Egypt dating to the period 4000 years ago. The disease is one of few that are recorded so early and at the same time the one that has been creating curing problems ever since till today (Kolasinski, 2014). As a result of some disorders in the uric acid metabolism, the deposition of its crystals takes place in tissues and organs (Hainer et al., 2014; Lamer-Zarawska et al., 2012; Majdan and Borys, 2010). It causes pain of joints and forming of tophi and tophi kidney.

The limit of uric acid in the blood is $6.5 \mathrm{mg} \%$. Above the value, the process of acid crystal forming takes place and the crystals gather in tissues, joints or urine ducts (taking the form of urates) (Lamer-Zarawska et al., 2012). As a result of the crystals cumulation in joints, the inflammatory states are activated and the joint is damaged.

The most frequently affected places are metatarsus joints, but also the joints of wrists, ankles, knees and fingers (Hainer et al., 2014). Three potential factors influencing the condition are mentioned here. Firstly, an increased level of uric acid in the blood may result from inhibition of purines production process. Moreover, if the resynthesis of purine nucleotides is decreased, a simultaneous increase of the uric acid in blood will be observed. Thirdly, a disturbed disposition/excretion of the uric acid via kidneys (Lamer-Zarawska et al., 2012) is mentioned. Gout is most frequently diagnosed based on the clinical criteria worked out by the American College of Rheumatology (Hainer et al., 2014).

\section{Epidemiology and factors facilitating the development of gout}

The frequency of gout occurrence increases along with the age (Hainer et al., 2014). The morbitity more frequently corresponds to men (Majdan and Borys, 2010; Raciborski et al., 2015) among whom the disease is the most frequent cause of joints inflammation (Raciborski et al., 2015). The frequency of gout occurrence in men and women is 6:1 (Gińdzieńska-Sieśkiewicz et al., 2010).

Women usually fall ill at 65, after the menopause, and then the occurrence frequency in both men and women is at a comparable level (Majdan and Borys, 2010). It is connected with an increased excretion of uric acid via female hormones (Hainer et al., 2014); in the post-menopausal period the level of estrogenes is lowered, bringing about an increase in the uric acid level in blood (Raciborski et al., 2015).

Worldwide the number of people suffering from gout is increasing (Majdan and Borys, 2010), which has been noticeable within the last 50 years (Kuo et al., 2015). Morbidity in the world (both old and new cases) is found at the level of $1.4-3.9 \%$ in the adult population (Raciborski et al., 2015). The Pacific region countries show the highest gout morbidity.

Taking into consideration all the world countries, the higher gout morbidity frequency is characteristic for the group of the highly developed states and the tendency is steadily growing (Kuo et al., 2015). The accessible literature data show that the incidence number of gout has grown by more than 200\% in the last decade (Gińdzieńska-Sieśkiewicz et al., 2010; Majdan, 2013). The incidence in the European countries is estimated at the annual level $1-2 \%$. The percentage of those suffering from gout in Poland, owing to a lack of precise data, is difficult to define; only Raciborski et al. (2015) prepared a questionnaire examination on whose basis it was stated that approx. 1\% 
Jurczak, R., Reguła, J. (2016). Plant materials used in supporting the treatment of gout. Nauka Przyr. Technol., 10, 4, \#58. DOI: http://dx.doi.org/10.17306/J.NPT.2016.4.58

of the population may suffer from the disease. Basing on the above, it is concluded that the number of citizens suffering from the disease may reach from 380 to 780 thousands (Raciborski et al., 2015). The development of the disease is conditioned by the age, susceptibility to metabolic diseases, including obesity (Majdan and Borys, 2010), metabolic syndrome, arterial hypertension, diabetes, coronary disease; moreover, application of some medicines, such as: loop diuretics and thiazides, cyclosporine, ethambutol, pyrazynamidum, low aspirin dosages (Raciborski et al., 2015), inadequate diet, abundant consumption of animal-origin foodstuffs, sea fruits, alcohol, and especially beer (Gibson, 2004; Zimmermann-Górska, 2006). Genetic factors, race and/or ethnic group also influence the incidence rate. Representatives of the black race, the Maori and Chinese are more susceptible than Europeans and/or the white race representatives (Singh, 2013). Genetic conditioning and improper protein-rich and red-meat abundant diet, excessive alcohol consumption, as well as overweight favour the development of primary gout which is always accompanied by co-occurring diseases, including arterial blood hypertension, as well as lipid and carbohydrates metabolism disorders. The secondary gout is caused by an increase and breakdown of nucleotides parallel to an increased cytolysis in case of some disorders, in haemolitic anaemia and tumour diseases (Lamer-Zarawska et al., 2012). Gout has long been associated with the lifestyle. It used to be called kings' disease, the phrase connected with the consumption of certain productsrich in purines (Table 1), including meat, giblets, sea fruits, breads, pastries and alcoholic drinks (Kolasinski, 2014). The consumption of fizzy beverages, sugar- and fructosesweetened juices, also favours the higher frequency of gout, both in women and men. It is worth noting here that applying an energy and carbohydrate-limited content diet, even if the level of purines-rich products in not reduced, favours the reduction of the risk of joints inflammation fits and the level of uric acid in blood. Moreover, the level of uric acid in blood is connected with the body mass and BMI (Body Mass Index) - the higher BMI value and lower muscle mass index, the higher probability of an increased level of uric acid in blood. There are reports informing about a favourable influence of the Mediterranean diet on the treatment of gout (Guasch-Ferré et al., 2013; Kolasinski, 2014). Moreover, it has been recently discovered that the level of uric acid in blood, as well as the degree of its excretion by the organism, is genetic polymorphism-dependent in SLC2A9.

The following are numbered among the factors causing susceptibility to gout:

- genetic conditioning (gene SLC2A9)

- hyperuricemia

- metabolic syndrome

- medicines (cyclosporine, diuretics, acetylsalicylic acid at low dosages)

- overweight and aortal hypertension

- meat and sea fruits-abundant diet

- excessive consumption of alcohol, especially beer

- organ transplants

- male sex and post-menopausal period in women (Le MyPhuong et al., 2008)

- operation or a recent tumour

- dehydration (Raciborski et al., 2015)

- old age (Gibson, 2004; Zhang et al., 2006). 
Jurczak, R., Reguła, J. (2016). Plant materials used in supporting the treatment of gout. Nauka Przyr. Technol., 10, 4, \#58. DOI: http://dx.doi.org/10.17306/J.NPT.2016.4.58

Table 1. Content of purines in some foods (Souci et al., 2008) - products of high and medium content of purines

Tabela 1. Zawartość puryn w wybranych produktach spożywczych (Souci i in., 2008) - produkty o wysokiej i średniej zawartości puryn

\begin{tabular}{|c|c|}
\hline Products - Produkty & $\begin{array}{l}\text { Purine content - Zawartość puryn } \\
\qquad(\mathrm{mg} / 100 \mathrm{~g})\end{array}$ \\
\hline Smoked sprat - Szprot wędzony & 804 \\
\hline Yeasts - Drożdże & 680 \\
\hline Pork heart - Serce wieprzowe & 530 \\
\hline Pork liver - Wątroba wieprzowa & 515 \\
\hline Sardine - Sardynka & 480 \\
\hline Calf liver - Wątroba cielęca & 460 \\
\hline Trout - Pstrąg & 297 \\
\hline Tuna - Tuńczyk & 257 \\
\hline Chicken liver - Wątroba z kurczaka & 243 \\
\hline Anchois & 239 \\
\hline Herring - Śledź & 219 \\
\hline Bean - Fasola & 200 \\
\hline Soy - Soja & 200 \\
\hline Halibut & 178 \\
\hline Chicken breast - Pierś z kurczaka & 175 \\
\hline Salmon - Łosoś & 170 \\
\hline Poppy seeds - Nasiona maku & 170 \\
\hline Carp - Karp & 160 \\
\hline Shrimps - Krewetki & 147 \\
\hline Pork joint with bone - Schab z kością & 145 \\
\hline Mackerel - Makrela & 145 \\
\hline Sunflower seeds - Nasiona słonecznika & 143 \\
\hline Duck breast - Pierś z kaczki & 138 \\
\hline Rabbit meat - Mięso królicze & 132 \\
\hline Ham - Szynka & 131 \\
\hline Sirloin, Neck, Fore Shank - Polędwica, karkówka, łopatka wołowa & 130 \\
\hline Lobster - Homar & 118 \\
\hline European pike-perch - Sandacz & 110 \\
\hline Pork sausage - Kiełbasa wieprzowa & 110 \\
\hline Pea - Groch & 109 \\
\hline Chick-pea - Ciecierzyca & 109 \\
\hline Grapes - Winogrona & 107 \\
\hline
\end{tabular}


Jurczak, R., Reguła, J. (2016). Plant materials used in supporting the treatment of gout. Nauka Przyr. Technol., 10, 4, \#58. DOI: http://dx.doi.org/10.17306/J.NPT.2016.4.58

\section{Treatment methods of gout and the importance of diet in weakening its symptoms}

The basic curing notions here are: lifestyle change, applying a specialist diet and appropriate physical activity. Gradual reduction of body mass is indispensable, as well as intensification of physical activity along with abandoning both alcoholic and sweetened drinks. Pharmacological treatment aiming at lowering of the level of uric acid in blood consists in applying allopurinol, alternatively uricosuric drugs and febuxostat. Low doses of colchicine (at max. $2 \mathrm{mg}$ per 1 day), NSAIDs or/and glucocorticosteroids are applied in case of gout attacks (Raciborski et al., 2015). The treatment applied is a three-direction procedure. The level of uric acid in blood needs to be lowered to the values acceptable to the organism, the acid crystals removed and the pain reduced. The treatment basically consists in lowering the hyperuricemia by inhibiting the production of the uric acid.

Apart from an appropriate diet, the basis of the treatment is lowering of the BMI, along with an application of medicines and diet supplements, most frequently of plantorigin (Lamer-Zarawska et al., 2012).

That is why the applied gout curing medicines are divided into 3 basic groups:

- medicines inhibiting xantine oxidase

- uricosuric medicines

- biological medicines inhibiting the activity of interleukin 1 (IL-1) (Raciborski et al., 2015).

Diet recommendations during gout treatment comprise among others the following indications: calorific diet, purines-rich products (Table 1) reduction, mainly those of animal-origin, including dairy and vitamin C-rich products. Consumption of low-calorie dairy products is connected with decreasing the concentration of uric acid in blood, as a result of its intensified excretion; the products provide lower content of purines and show an anti-inflammatory activity.

Then, providing vitamin $\mathrm{C}$ in the diet of gout patients may decrease the concentration of uric acid in blood, increasing urine excretion and decreasing the content of uric acid in kidneys as a result of the presence of competitive bonds. Application of raw plant materials is widely described in the traditional Chinese (TCM) and ajurvedic medicine, where herb mixtures for gout patients are recommended (Kolasinski, 2014).

\section{Raw plant materials utilised in gout treatment}

Utilisation of raw plant materials is basically focused on their analgesic and antiinflammatory and diuretic activity (Table 2). The following plants, or their parts, containing anti-inflammatory substances are used: plant salicylates, willow bark, Flos $\mathrm{Ul}$ mariae, Harpagophytum procumbens DC, Rosa canina (Lamer-Zarawska et al., 2012), sour cherry (Prunus cerasus L.) and its products (Zhang et al., 2012), Aegopodium podagraria L. (Trąba et al., 2012).

Colchicine - a powerful toxic alkaloid isolated from Colchicum autumnale - is applied in severe pain attacks. Then, diuretic activity plants, removing an excess of urates 
Jurczak, R., Reguła, J. (2016). Plant materials used in supporting the treatment of gout. Nauka Przyr. Technol., 10, 4, \#58. DOI: http://dx.doi.org/10.17306/J.NPT.2016.4.58

Table 2. Plants supporting treatment of gout

Tabela 2. Rośliny wspomagające leczenie dny moczanowej

\begin{tabular}{|c|c|}
\hline $\begin{array}{l}\text { Plant's name and medici- } \\
\text { nal properties part } \\
\text { Nazwa rośliny oraz część } \\
\text { wykazująca działanie } \\
\text { lecznicze }\end{array}$ & $\begin{array}{l}\text { Biologically active compounds (chemical composition) and medicinal activity } \\
\text { Związki biologicznie czynne (skład chemiczny) oraz działanie lecznicze }\end{array}$ \\
\hline 1 & 2 \\
\hline $\begin{array}{l}\text { Betula pendula } \text { Roth } \\
\text { Brzoza brodawkowata } \\
\text { (Nartowska, 2011) }\end{array}$ & $\begin{array}{l}\text { Flavonoid compounds-flavonols: quercetin glycosades (hyperoside, quercetin, } \\
\text { rutoside, 3-arabinofuranoside, 3-rabinopyranoside, 3-quercetin rhamnoside), } \\
\text { myricetin (3-0-galactodide of myricetin and kaempferol), } \\
\text { flavone-glycosades of luteolin, katechin tannins, organic acids (ascorbic, chloro- } \\
\text { genic, caffeic and p-coumarin acids), triterpenes of dammarane and lupane type } \\
\text { (free and ester-bound with malonic and acetic acids), polysaccharides, } \\
\text { mineral elements (K, Mn, Ni, Zn), } \\
\text { volatile oil with sesquiterpenes of a-butulenol and acetate of a-butulenol } \\
\text { Effects: diuretic, removes chloride and sodium ions } \\
\text { Związki flawonoidowe - flawonole: glikozydy kwercetyny (hiperozyd, kwercyty- } \\
\text { na, rutozyd, 3-arabinofuranozyd, 3-arabinopiranozyd, 3-ramnozyd kwercetyny), } \\
\text { miricetyny, 3-0-galaktozyd miricetyny i kemferolu), } \\
\text { flawony - glikozydy luteoliny, garbniki katechinowe, kwasy organiczne, kwas } \\
\text { askorbinowy, kwas chlorogenowy, kwas kawowy, kwas p-kumarowy), triterpeny } \\
\text { typu dammaranu i lupanu (wolne i w związkach estrowych z kwasem malono- } \\
\text { wym, kwasem octowym), polisacharydy, } \\
\text { sole mineralne (K, Mn, Ni, Zn), } \\
\text { olejek eteryczny wraz z zawartością seskwiterpenów a-betulenolu i octanu } \\
\text { a-betulenolu } \\
\text { Działanie: Moczopędne, usuwa jony chlorkowe i sodowe }\end{array}$ \\
\hline $\begin{array}{l}\text { Rosa canina } \mathrm{L} . \\
\text { Dzika róża } \\
(\text { Grys, 2009) }\end{array}$ & $\begin{array}{l}\text { Poliphenols (flavonoids, proanthocyanidines), } \\
\text { organic acids (L-ascorbic and dehydroxyascorbic acids), } \\
\text { GOPO (galactolipid), } \\
\text { volatile oil, } \\
\text { EFA (linoleic and a-linoleic, oleic, palmityne and stearic acids), } \\
\text { mineral elements (Na, K, } \mathrm{Ca}, \mathrm{P}, \mathrm{Mg}, \mathrm{S}, \mathrm{Fe}, \mathrm{Cu}, \mathrm{Zn}, \mathrm{Mn}, \mathrm{Al} \text { ), } \\
\left.\text { vitamins: } \mathrm{B}_{1}, \mathrm{~B}_{2}, \mathrm{PP}, \mathrm{A}, \mathrm{E}, \mathrm{K}\right) \\
\text { Effects: tonic to immunological system, anti-tumour, energizing the organism, } \\
\text { antioxidative, anti-inflammatory, diuretic, calming, anti-migraine, anti-septic, anti- } \\
\text { bacterial, providing elasticity to skin } \\
\text { Polifenole (flowonoidy, proantocyjanidyny), } \\
\text { kwasy organiczne (kwas L-askorbinowy i kwas dehydroksyaskorbowy), } \\
\text { GOPO (galaktolipid), } \\
\text { olejek eteryczny, } \\
\text { NNKT (kwas linolowy i a-linolenowy, oleinowy, palmitynowy i stearynowy), } \\
\text { składniki mineralne (Na, K, Ca, P, Mg, S, Fe, Cu, Zn, Mn, Al), } \\
\text { vitamins: } \mathrm{B}_{1}, \mathrm{~B}_{2}, \mathrm{PP}, \mathrm{A}, \mathrm{E}, \mathrm{K} \text { ) } \\
\text { Działanie: Wzmacnianie układu odpornościowego, działanie przeciwnowotworo- } \\
\text { we, działanie ogólnie wzmacniające na organizm, działanie antyoksydacyjne, } \\
\text { przeciwzapalne, moczopędne, uspokajające, przeciwmigrenowe, antyseptyczne, } \\
\text { antybakteryjne, wpływ na uelastycznianie skóry }\end{array}$ \\
\hline
\end{tabular}


Jurczak, R., Reguła, J. (2016). Plant materials used in supporting the treatment of gout. Nauka Przyr. Technol., 10, 4, \#58. DOI: http://dx.doi.org/10.17306/J.NPT.2016.4.58

Table 2 - cont. / Tabela 2 - cd.

\begin{tabular}{|c|c|}
\hline 1 & 2 \\
\hline $\begin{array}{l}\text { Viola tricolor } \\
\text { Fiołek trójbarwny } \\
\text { (Jambor, 2012) }\end{array}$ & $\begin{array}{l}\text { Flavonoids (rutoside, O-glycosades of quercetine), } \\
\text { C-glycosades (vitexin, saponaretine, orientine, zoorentine), } \\
\text { salicylic acids derivatives (salicylic acid methylic ester, and glycosade of salicylic } \\
\text { acid methylic ester - violutoside) } \\
\text { Effects: diuretic } \\
\begin{array}{l}\text { Flawonoidy (rutozyd, O-glikozydy kwercetyny, C - glikozydy (witeksyna, sapona- } \\
\text { retyna, orientyna, zoorientyna) } \\
\text { pochodne kwasu salicylowego (ester metylowy kwasu salicylowego glikozyd estru } \\
\text { metylowego kwasu salicylowego wiolutozyd) } \\
\text { Działanie: moczopędne }\end{array}\end{array}$ \\
\hline $\begin{array}{l}\text { Harpagophytum } \\
\text { procumbens DC } \\
\text { Hakorośl rozesłana } \\
\text { (Wolski et al., 2010) } \\
\text { Root (tubers) } \\
\text { Bulwy spichrzowe } \\
\text { (korzeń) }\end{array}$ & 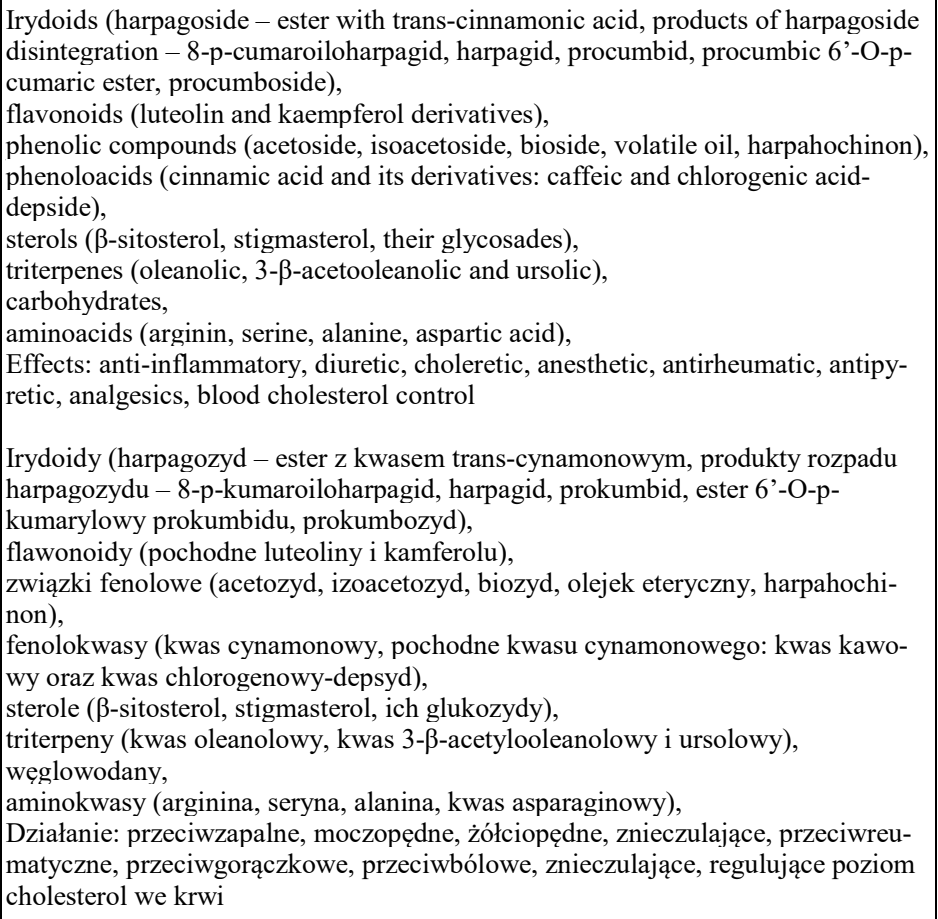 \\
\hline $\begin{array}{l}\text { Solidago virgaurea } \\
\text { Nawłoć pospolita } \\
\text { (Jambor, 2012) }\end{array}$ & $\begin{array}{l}\text { Flavonoids (quercetin, isoquercetin, kaempferol and their glycosades), } \\
\text { saponins (acidic and neutral reaction: tridesmosides and acetylene bidesmosides), } \\
\text { phenoloacids and their ester and glycosades compounds (phenolic diglycosade - } \\
\text { leicarposide), } \\
\text { volatile oil } \\
\text { Effects: diuretic, anti-inflammatory, analgesic } \\
\\
\text { Flawonoidy (kwercetyna, izokwercetyna, kemferol i ich glikozydy), } \\
\text { saponiny (kwaśne i obojętne: tridesmosydy i acetylowane bidesmosydy), } \\
\text { fenolokwasy i ich związki estrowe i glikozydowe (diglikozyd fenolowy - lejokar- } \\
\text { pozyd), } \\
\text { olejek eteryczny } \\
\text { Działanie: moczopędne, przeciwzapalne, przeciwbólowe }\end{array}$ \\
\hline
\end{tabular}


Jurczak, R., Reguła, J. (2016). Plant materials used in supporting the treatment of gout. Nauka Przyr. Technol., 10, 4, \#58. DOI: http://dx.doi.org/10.17306/J.NPT.2016.4.58

Table 2 - cont. / Tabela 2 - cd.

\begin{tabular}{|c|c|}
\hline 1 & 2 \\
\hline $\begin{array}{l}\text { Agropyron repens } \\
\text { Perz właściwy } \\
\text { (Jambor, 2012) }\end{array}$ & $\begin{array}{l}\text { Polisaccharides, basically fructans (tritycine), } \\
\text { monosaccharides (fruktose), } \\
\text { sugar alcohols (mannitol, inositol), } \\
\text { oligosaccharides, } \\
\text { mucuses, } \\
\text { volatile oil (carvone, capilene), } \\
\text { silicon compounds } \\
\text { Effects: prevention of kidney stones disease, gives elasticity to blood vessels walls } \\
\text { and connective tissue } \\
\text { Polisacharydy, głównie fruktany (trytycyna) } \\
\text { monosacharydy (fruktoza) } \\
\text { alkohole cukrowe mannitol, inozytol) } \\
\text { oligosacharydy } \\
\text { śluzy } \\
\text { olejek eteryczny (karwon, kapilen), } \\
\text { zwiazzki krzemu } \\
\text { Działanie: zapobiega wytwarzaniu kamieni nerkowych, uelastycznia ścianę naczyń } \\
\text { krwionośnych i tkankę łączną }\end{array}$ \\
\hline $\begin{array}{l}\text { Aegopodium podagraria } \\
\text { L. } \\
\text { Podagrycznik pospolity } \\
\text { (Kunstman et al., 2012) }\end{array}$ & $\begin{array}{l}\text { Phenolic compounds from coumarin group, flavonoids and phenolic acids, } \\
\text { organic acids (caffeic and chlorogenic acids), } \\
\text { glycosades, } \\
\text { quercetin derivatives: hiperoside (3-O-galactoside) and kaempferol: tripholine } \\
\text { (3-O-galactoside), } \\
\text { polyacetylene (polyynes): falcarinol (panaxynol, cis-heptadeca-1,9-diene-4,6- } \\
\text { diyno-3-ol), } \\
\text { falcarindiol (cis-heptadeca-1,8-diene-4,6-diyno-3,10-diol), } \\
\text { falcarinon (cis-heptadeca-1,9-dieno-4,6-diyno-3-on), } \\
\text { falkarinolon (cis-heptadeca-1,9-dieno-4,6-diyno-9-ol-3-on), } \\
\text { volatile oil, containing (basically) mono- and sesquiterpenes } \\
\text { Effects: anti-tumour, anti-inflammatory, anti-microbial } \\
\text { Związki fenolowe z grupy kumaryn, flawonoidów i kwasów fenolowych, } \\
\text { kwasy organiczne (kwas kawowy i kwas chlorogenowy) } \\
\text { glikozydy flawonolowe, } \\
\text { pochodne kwercetyny: hiperozyd (3-O-galaktozyd) i kemferolu: trifolina (3-O- } \\
\text { galaktozyd), } \\
\text { poliacetyleny (poliiny): farkalinol (panaksynol), cis-heptadeka-1,8-poliacetyleny } \\
\text { (poliiny): falkarinol (panaksynol, cis-heptadeka-1,9-dieno-4,6-diino-3-ol), falka- } \\
\text { rindiol (cis-heptadeka-1,8-dieno-4,6-diino-3,10-diol), falkarinon (cis-heptadeka- } \\
\text { l,9-dieno-4,6-diino-3-on), falkarinolon (cis-heptadeka-1,9-dieno-4,6-diino-9-ol-3- } \\
\text { on), } \\
\text { olejek eteryczny, który zawiera głównie mono- i seskwiterpeny } \\
\text { Działanie: przeciwnowotworowe, przeciwzapalne, przeciwdrobnoustrojowe }\end{array}$ \\
\hline $\begin{array}{l}\text { Urtica dioica } \text { L. } \\
\text { Pokrzywa zwyczajna } \\
\text { (Pieszak and } \\
\text { Mikołajczak, 2010) }\end{array}$ & $\begin{array}{l}\text { Flavonoids (kempferol, astragalin, rutin, isorhamnetin), anthocyanins, quercetin, } \\
\text { acids: caffeic, butyric, formic, coumaric, palmitic, succinic, pantotenoic, neo- } \\
\text { chlorogenic, oxalic, acetic, phosphoric, caffeic/ilo/malic, glycolic, linoleic, lino- } \\
\text { lenic, } \\
\text { organic compounds (chlorophyll, leucotrienes, ceramides, sphingosine, terpenes, } \\
\text { isolectins, 2-metyloheptanone (volatile compounds), monoterpendioles, glyco- } \\
\text { sades of monoterpendioles, histamine, serotonine, acetylocholine, lecitin, lectine, } \\
\text { scopolectin, coproporphirine, agglutinin, acetophenon, alkaloids), }\end{array}$ \\
\hline
\end{tabular}


Jurczak, R., Reguła, J. (2016). Plant materials used in supporting the treatment of gout. Nauka Przyr. Technol., 10, 4, \#58. DOI: http://dx.doi.org/10.17306/J.NPT.2016.4.58

Table 2 - cont. / Tabela 2 - cd.

\begin{tabular}{|c|c|}
\hline 1 & 2 \\
\hline & $\begin{array}{l}\text { sterole ( } \beta \text {-sitosterol, stigmasterol, kaempfesterol), } \\
\text { carotenoids: xanthphyll, violaxanthin, } \\
\text { vitamins: A, C, E, K, B2, pantotenoic acid, folacin, cholin, } \\
\text { mineral components (Ca, Fe, Mg, Si), } \\
\text { monosaccharides and oligosaccharides (glucose, fructose, galactose, myo-inositol, } \\
\text { raffinose, maltose), } \\
\text { polisaccharides (arabinogalactans, glucan, glucogalacturonic), } \\
\text { tannines, } \\
\text { lignans } \\
\text { Effects: Intensifies water diuresis, supports the immunological system, acts anti- } \\
\text { inflammatorily, analgesic, lowers the level of cholesterol, blood pressure, urea and } \\
\text { sodium chloride, reduces hair loss } \\
\text { Flawonoidy (kemferol, astragalina, rutyna, izoramnetyna), antocyjany, kwercetyna } \\
\text { kwasy: kwas kawowy, masłowy, mrówkowy, kumarowy, bursztynowy, } \\
\text { palmitynowy, pantotenowy, neochlorogenowy, szczawiowy, octowy, fosforowy, } \\
\text { kawoilojabłkowy, glikolowy, linolowy, linolenowy } \\
\text { związki organiczne (chlorofil, leukotrieny, ceramidy, sfingozyna, terpeny, izolek- } \\
\text { tyny, 2-metyloheptanon (związki lotne), onoterpendiole, } \\
\text { glikozydy monoterpendioli, histamina, serotonina, acetylocholina, lecytyna, lekty- } \\
\text { ny, skopolektyna, koproporfiryna, aglutynina, } \\
\text { acetofenon, alkaloidy), } \\
\text { sterole ( } \beta \text {-sitosterol, stigmasterol, kampesterol), } \\
\text { karotenoidy: ksantofil, violaksantyna } \\
\text { witaminy: A, C, E, K, B, kwas pantotenowy, folacyna, cholina, } \\
\text { składniki mineralne (wapń, żelazo, magnez, krzem), } \\
\text { monosacharydy i oligosacharydy (glukoza, fruktoza, galaktoza, mio-inozytol, } \\
\text { rafinoza, maltoza), } \\
\text { polisacharydy (arabinogalaktany, glukan, glukogalakturoniany), garbniki (taniny), } \\
\text { lignany } \\
\text { Działanie: wzmaga diurezę wodą, wspomaganie układu odpornościowego, działa- } \\
\text { nie przeciwzapalne, przeciwbólowe, obniża poziom cholesterol we krwi, obniża } \\
\text { poziom ciśnienia krwi, mocznika i chlorku sodu, ogranicza wypadanie włosów }\end{array}$ \\
\hline $\begin{array}{l}\text { Equisetum arvense L. } \\
\text { Skrzyp polny } \\
\text { (Cisowski, 2010) }\end{array}$ & $\begin{array}{l}\text { Flavonoids (flavonols derivatives, kaempferol and quercetin glycosades: isoquer- } \\
\text { citrin (isoquercitroside) quercetin - 3-glucoside and its malonic acid ester binding, } \\
\text { equiscetrin - kaempferol-7-diglucoside, quercetin-3-sophoroside, flavon deriva- } \\
\text { tives: protogenkwanin-4-glucoside, genkwanin - apigenin-7-methyl ester, phenolic } \\
\text { acids (caffeic and protocatechuic acid methyl ester, chlorogenic and dikawoilowi- } \\
\text { nowy acids), } \\
\text { mineral compounds (silicon, aluminum, magnesium, potassium), } \\
\text { phytosterol (ß-sitosterol, campestrol, isofucosterol, trace cholesterol), } \\
\text { polyenoic acid } \\
\text { ascorbic acid, } \\
\text { saccharum (glucose, fructose, saccharose, lactose, sugar alcohols: mannitol, inositol), } \\
\text { lectines (glycoproteids), } \\
\text { beta-D-glucan } \\
\text { Effects: diuretic, weakly anti-bacterial and anti-inflammatory, remineralizing, anti- } \\
\text { oxidative }\end{array}$ \\
\hline & $\begin{array}{l}\text { Flawonoidy (pochodne flawonolowe, glikozydy kemferolu i kwercetyny: izokwer- } \\
\text { cytryna (izokwercytrozyd) - 3-glukozyd kwercetyny i jej połączenia estrowe } \\
\text { z kwasem malonowym, }\end{array}$ \\
\hline
\end{tabular}


Jurczak, R., Reguła, J. (2016). Plant materials used in supporting the treatment of gout. Nauka Przyr. Technol., 10, 4, \#58. DOI: http://dx.doi.org/10.17306/J.NPT.2016.4.58

Table 2 - cont. / Tabela 2 - cd.

\begin{tabular}{|c|c|}
\hline 1 & 2 \\
\hline & $\begin{array}{l}\text { ekwizetryna-7-diglukozyd kemferolu, 3-soforozyd kwercetyny, pochodne flawo- } \\
\text { nu: 4-glukozyd protogenkwaniny, genkwanina - 7-metylowy eter apigeniny), } \\
\text { fenolokwasy (ester metylowy kwasu kawowego i kwasu protokatechowego, kwas } \\
\text { chlorogenowy, kwas } \\
\text { dikawoilowinowy), } \\
\text { związki mineralne (krzem, glin, magnez, potas), } \\
\text { fitosterole (ß-sitosterol, kampestrol, izofukosterol, w śladowych ilościach choleste- } \\
\text { rol), } \\
\text { kwasy polienowe, } \\
\text { kwas askorbowy, } \\
\text { cukrowce (glukoza, fruktoza, sacharoza, laktoza, alkohole cukrowe: } \\
\text { mannitol, inozytol), } \\
\text { lektyny (glikoproteidy), } \\
\text { beta-D-glucan } \\
\text { Działanie: moczopędne, przeciwbakteryjne i przeciwzapalne, remineralizujące, } \\
\text { przeciwutleniające }\end{array}$ \\
\hline $\begin{array}{l}\text { Filipendula ulmaria } \\
\text { Wiązówka błotna } \\
\text { (Smolarz and Sokołow- } \\
\text { ska-Woźniak, 2001) } \\
\text { Whole plant } \\
\text { Cała roślina }\end{array}$ & $\begin{array}{l}\text { Flavonoids (rutoside, hyperoside, quercetin-3-glucuronide, spiraeoside, quercetin- } \\
\text { 4'-glycoside and kaempferol, monotropitoside, heteroside), traces of coumarins, } \\
\text { phenolic glycosides (isosalicin, monotropitoside (gaultherin)) } \\
\text { tannins, } \\
\text { volatile oils (vanillin, methyl salicilan, salicylaldehyde, heliotropin), } \\
\text { organic acids (salicylic acid, salicylaldehyde, citric, and ascorbicum and ascorbini- } \\
\text { cum acids) } \\
\text { Effects: diaphoretic, antipyretic, antibacterial, antiviral, anti-inflammatory and } \\
\text { analgesic, antirheumatic, diuretic-cholagogue, astringent, antiulcerous, anti- } \\
\text { tumour, immunostimulating, dilates blood vessels, and decreases blood viscosity } \\
\text { Flawonoidy (rutozyd, hiperozyd, 3-glukuronidu kwercetyny, spiraeozydu, } \\
\text { 4'-glikozydu kwercetyny i kemferolu, monotropitozyd, } \\
\text { heterozyd), ślady kumaryn, } \\
\text { glikozydy fenolowe (izosalicyna, monotropitozyd (gaulteryna)) } \\
\text { garbniki, } \\
\text { olejki eteryczne (wanilina, salicylan metylu, aldehyd salicylowy, heliotropina), } \\
\text { kwasy organiczne (kwas salicylowy, aldehyd salicylowy, kwas } \\
\text { cytrynowy, kwas askorbowy), } \\
\text { Działanie: napotne, przeciwgorączkowe, przeciwbakteryjne, przeciwwirusowe, } \\
\text { przeciwzapalne, przeciwbólowe, przeciwreumatyczne, moczopędne, żółciopędne, } \\
\text { ściągające, przeciwwrzodowe, przeciwnowotworowe, immunostymulacyjne, } \\
\text { rozszerza naczynia krwionośne i zmniejsza lepkość krwi }\end{array}$ \\
\hline $\begin{array}{l}\text { Salix alba L. } \\
\text { Wierzba biała } \\
\text { (Michalska and Pham, } \\
\text { 2002) }\end{array}$ & $\begin{array}{l}\text { Salicylic glycosides (salicine, salicortin, populin, alireposide, salidroside, } \\
\text { 3-O-cynamoilosalicine) } \\
\text { Effects: analgesic, anti-inflammatory, antipyretic } \\
\text { Glikozydy salicylowe (salicyna, salikortyna, populina, salirepozyd, } \\
\text { salidrozyd, 3-O-cynamoilosalicyna) } \\
\text { Działanie: przeciwbólowe, przeciwzapalne, przeciwgorączkowe }\end{array}$ \\
\hline $\begin{array}{l}\text { Ononis spinosa } \\
\text { Wilżyna ciernista } \\
\text { (Jambor, 2012) }\end{array}$ & $\begin{array}{l}\text { Flawonoidy (isoflavons: ononin, (formononetin-7-glucoside), formononetin, } \\
\text { genistin), } \\
\text { volatile oil (trans-anetol, carvon, menthol) } \\
\text { Effects: diuretic }\end{array}$ \\
\hline
\end{tabular}


Jurczak, R., Reguła, J. (2016). Plant materials used in supporting the treatment of gout. Nauka Przyr. Technol., 10, 4, \#58. DOI: http://dx.doi.org/10.17306/J.NPT.2016.4.58

Table 2 - cont. / Tabela 2 - cd.

\begin{tabular}{|c|c|}
\hline 1 & 2 \\
\hline $\begin{array}{l}\text { Root } \\
\text { Korzeń }\end{array}$ & $\begin{array}{l}\text { Flawonoidy (izoflawony: ononina, (7-glukozyd formononetyny), } \\
\text { formononetyna, genistyna), } \\
\text { olejek eteryczny (trans-anetol, karwon, mentol) } \\
\text { Działanie: moczopędne }\end{array}$ \\
\hline $\begin{array}{l}\text { Prunus cerasus L. } \\
\text { Wiśnia pospolita } \\
\text { (McCune et al., 2011; } \\
\text { Kolasiński, 2014) }\end{array}$ & $\begin{array}{l}\text { Flavonoids: antocyanins, quercetin, } \\
\text { hydroxycynamonian, } \\
\text { mineral elements: potassium, } \\
\text { vitamins: C, carotenoids, } \\
\text { melatonin, } \\
\text { fiber } \\
\text { Effect: anti-inflammatory, anti-oxidative, reduces uric acid level } \\
\text { Flawonoidy: antocyjany, kwercetyna, } \\
\text { hydroksycynamonian, } \\
\text { składniki mineralne: potas, } \\
\text { witaminy: witamina C, karotenoidy, } \\
\text { melatonina, } \\
\text { błonnik } \\
\text { Działanie: przeciwzapalne, antyoksydacyjne, obniża poziom kwasu moczowego }\end{array}$ \\
\hline
\end{tabular}

comprise e.g.: Harpagophytum procumbens DC, Flos Ulmariae, Stigma Maydis, Folium Urticae, Rosa canina, Pericarpium Phaseoli, Radix Ononidis, Rhizoma Agropyri, Herba Polygoni avicularis (Lamer-Zarawska et al., 2012), Folium Betulae (Solidago virgaurea) (Błecha and Wawer, 2011), Aegopodium podagraria L., Equisetum arvense.

\section{Aegopodium podagraria $\mathrm{L}$.}

It is a plant occurring in Europe, Asia Minor, Siberia and the Caucasus, as well as in south America (Trąba et al., 2012). It has been present in the folk medicine since the antiquity, utilised for curing numerous rheumatic diseases - sciatica and gout. Both the herb and the roots are used as curing means, but in the old days the plant was used as a vegetable (Kunstman et al., 2012).

Young leaves and shoots were an element of numerous dishes, including salads or soups, as a source of precious nutritional values (Trąba et al., 2012). Nowadays, dried leaves are applied to prepare infusions for gout treatment, as well as haemorrhoids, inflammation of the bladder and kidneys. It is also used adjuvantly for renal calculi (Kunstman et al., 2012; Sarwa, 2001). Such infusions act slightly anti-inflammatorily, uretically and analgesically. Moreover, they favourably influence the metabolism, controlling the rate (Stefanovic et al., 2009). The anti-inflammatory activity is conditioned by the presence of polyacetylenes. The conducted in vitro test showed a 30-fold stronger activity of the compounds compared to indomethacin (a curing anti-inflammatory and anti-arthritis means) (Priori et al., 2007).

Falcarinol - is a compound present in Aegopodium podagraria L., yet it presents neurotoxic activity. However, no toxicity of the compound has been reported so far. Side effects can be observed only after very strong doses. Excessive doses can also provoke an allergic reaction (Christensen and Brandt, 2006). 
Jurczak, R., Reguła, J. (2016). Plant materials used in supporting the treatment of gout. Nauka Przyr. Technol., 10, 4, \#58. DOI: http://dx.doi.org/10.17306/J.NPT.2016.4.58

\section{Harpagophyti radix}

The plant originates from south Africa and it has been long applied by the tribal medicine to cure many diseases, including gastro-intestinal problems, bone-articular pains and spine problems (Lamer-Zarawska et al., 2012). It appeared in Europe in the mid XXth century (Lis, 2010), after detailed pharmacological and phytochemical examinations (Lamer-Zarawska et al., 2012).

A test on rats proved that a water extract of Harpagophyti radix shows anti-inflammatory, analgesic, and additionally anti-diabetic activity, lowering the level of sugar in blood (Mahomed and Ojewole, 2004). The active component in the plant is, first of all, harpagoside (Lis, 2010), as well as beta-sitosterol (Lamer-Zarawska et al., 2012). The therapeutic activity of the plant root runs at a few levels, first of all showing anti-inflammatory, analgesic (Andersen et al., 2004; Błecha and Wawer, 2011; ESCOP, 2009; Lamer-Zarawska et al., 2012; Lis, 2010) and anti-proliferative properties (Błecha and Wawer, 2011; Lamer-Zarawska et al., 2012).

It could satisfactorily replace an application of non-steroid anti-inflammatory medicines (Lis, 2010), e.g. phenylobuthazone, in many cases. The root's activity is compared to the one of such medicines as: phenylobuthazone or cortisone (Lamer-Zarawska et al., 2012). Moreover, owing to the presence of plant phenols and flavonoids, the plant shows antioxidative (Lis, 2010) and regulatory activity, improving both metabolism (Denner, 2007) and digestion (Matławska, 2006).

It strengthens the process of bil and digestion juices excretion (Lamer-Zarawska et al., 2012; Lis, 2010), lowers the level of blood cholesterol and shows anti-arthritis activity (Lis, 2010). Owing to the presence of flavonoids its duretic activity is performed effectively influencing an increase in the uric acid excretion. The active substances, present in the root of the plant, in rheumathoid disorders bring about weakening of the disorder's symptoms, improvement in the joints condition, increase in their mobility, reduction of the articular cartilage, and simultaneously they speed up the process of the joints regeneration. Moreover, they influence the regulation of the levels of both uric acid and bilirubin in blood (Lamer-Zarawska et al., 2012). Harpagophyti radix interacts with anti-arythmic medicines and those applied in hypertension, making their activity stronger. Application of the harpagophytum procubens shows an activity of a relatively low toxicity (Al-Harbi et al., 2013). The daily dose of the root while supporting rheumathoid disorders treatment is $4.5 \mathrm{~g}$ of the raw material (Lamer-Zarawska et al., 2012).

\section{Salicis cortex}

Willows are trees growing in Europe, Asia and North Africa (Lamer-Zarawska et al., 2012). The medicinal material is dried white or purple willow bark (Lamer-Zarawska et al., 2012; Trąba et al., 2012), collected before the appearance of first spring leaves (Lamer-Zarawska et al., 2012; Trąba et al., 2012). It was already in the antiquity (Struczyński, 2004) that the willow bark was applied in treating rheumathoid disorders. It was used both against rheumatism, and fever or pains (Trąba et al., 2012). The active compounds in willow bark are salicin glycosides (e.g. salicin, salicortin, salireposide, populina), flavonoids, phenolic acids (e.g. salicylic and p-coumaric acids) and tannins. The willow bark owes its healing capacity, first of all, to salicins (Lamer-Zarawska et 
Jurczak, R., Reguła, J. (2016). Plant materials used in supporting the treatment of gout. Nauka Przyr. Technol., 10, 4, \#58. DOI: http://dx.doi.org/10.17306/J.NPT.2016.4.58

al., 2012). Yet, the compounds were isolated from plants as late as the beginning of the XIXth c. The bark shows both analgesic, anti-inflammatory and antipyretic activity (Błecha and Wawer, 2011). The presence of tannins and flavonoids also intensifies the anti-inflammatory activity of the bark (Struczyński, 2004). It was demonstrated in animals' tests that isolated willow bark extract had an anti-inflammatory effect comparable to the one of the acetylsalicylic acid (Kahkonen et al., 1999). Moreover, flavonoids act diuretically and anti-oxidatively, and may parallely also strengthen the activity of salicylates (Lamer-Zarawska et al., 2012).

Nowadays, the willow bark is utilised in numerous diseases, including rheumathoid disorders, insomnia, neuralgia, colds, as well as gastroenteritis, or diarrhoea (Trąba et al., 2012). Mills et al. (1996) carried out 2-months long experiments with a double blind test on 82 patients with chronic inflammation of joints. The patients were randomly divided into 2 groups - the first one was given the willow bark preparation, while the other placebo. A mild decrease of patients' pain symptoms with a simultaneous absence of side-effects of the willow bark aided therapy was demonstrated (Mills et al., 1996). The willow bark substances should not be applied alongside with non-steroid antiinflammatory medicines, e.g. aspirin, since they may get intensified (Lamer-Zarawska et al., 2012). Willow bark decoctions made from 6-12 $\mathrm{g}$ of the material show both antipyretic and anti-inflammatory effect (Lamer-Zarawska et al., 2012).

\section{Betulae folium}

Birch leaves are a phytotherapeutic raw material obtained from Verrucosae and white-bark birch. The leaves are collected in early spring, and next dried in a natural way. The medicinal substances they contain are: flavonoids, catechin tannins, saponins, triterpens, polyprenols, as well as essential oils (Lamer-Zarawska et al., 2012). Moreover, young leaves contain resin and organic acids. Birch leaves demonstrate the diaphoretic (Trąba et al., 2012), diuretic (Błecha and Wawer, 2011; ESCOP, 2003), and antitumid activity, increasing $\mathrm{Na}+, \mathrm{Cl}$ - (Lamer-Zarawska et al., 2012) and uric acid excretion along with urine (Matławska, 2006). Moreover, applying the leaves will regulate the metabolism and detoxicate the organism (Lamer-Zarawska et al., 2012). The diuretic activity of the leaves is achieved at $6-9 \mathrm{~g} / 24 \mathrm{~h}$ of the material, with a single dose of $2-3 \mathrm{~g}$ (Lamer-Zarawska et al., 2012).

\section{Flos Ulmariae}

Mud meadowsweet flower contains numerous active substances including phenolic glycosides, essential oils, tannins and flavonoids (Lamer-Zarawska et al., 2012). The presence of salicyl acid, as well as phenolic compounds (salicylaldehyde and salicin), makes the flower act analgesically and anti-inflammatorily (Matławska, 2006; Struczyński, 2004). Moreover, diaphoretic, anti-pyretic, cholagogue and diuretic activities are observed, along with constrictive and anti-bacterial ones. It is also used in temperature-accompanied diseases, muscle pains and rheumathoid disorders, especially in patients suffering from kidney problems and ulcer diseases of both duodenum and stomach (Lamer-Zarawska et al., 2012). Flower infusions and decoctions are applied in joint inflammatory conditions (Struczyński, 2004). The compounds contained in meadowsweet enter into reactions with synthetic salicyl medicines (Lamer-Zarawska et al., 
Jurczak, R., Reguła, J. (2016). Plant materials used in supporting the treatment of gout. Nauka Przyr. Technol., 10, 4, \#58. DOI: http://dx.doi.org/10.17306/J.NPT.2016.4.58

2012). Liapina and Koval'chuk (1993) carried out a comparative test of meadowsweet flower and seed extract activity and stated that both the flowers and seeds show a high level of anti- thrombotic and fibrinolytic activity, when administered orally. Both the water and ethanol extract of meadowsweet shows an anti-oxidative activity. The extract also proved its hepatoprotective properties (Shilova et al., 2006) in the carried out mice test, in which the animals were stomach-administered $100 \mathrm{mg}$ per $1 \mathrm{~kg}$ of the extract for 5 days. The indicated therapeutic dose for adults is $2-6 \mathrm{~g}$ of meadowsweet flower. It is adviseable to drink the infusion a few times daily; the maximum dose 2.5-3.5 $\mathrm{g}$ of flowers or 4-5 $\mathrm{g}$ of the herb (Lamer-Zarawska et al., 2012).

\section{Sour cherries}

While treating gout, sour cherries and the fruits extracts are among the raw plant materials that should be, first of all, paid attention to as the source of anti-inflammatorily (Jacob et al., 2003; Kolasinski, 2014; Raciborski et al., 2015; Wang et al., 1999; Zhang et al., 2012) and anti-oxidatively acting anthocyanins (Blando et al., 2004; Kolasinski, 2014).

Moreover, the fruits may have an impact on decreasing the level of uric acid in blood (Jacob et al., 2003; Raciborski et al., 2015; Zhang et al., 2012), owing to the content of hydroxycinnamate and the earlier mentioned anthocyanins (Raciborski et al., 2015; Zhang et al., 2012).

A group of 663 people suffering from gout were subjected to tests with a sour cherries-enriched diet. It turned out after 2 days that the risk of gout attacks was lowered by $35 \%$ in the tested group of patients compared to those not given the diet. Similar effects were observed in the case of sour cherries extracts application. What is more, the application of the fruits acts favourably and helps in gout curing independently of the gender, level of obesity, quantity of consumed purines, level of alcohol consumption, as well as taking diuretic and anti-arthritis medicines. Owing to the application of a combined pharmacological treatment and participation of allopurinol with an addition of cherries or their extracts in the patients' diet, the risk of gout attacks decreased by $75 \%$ (Le MyPhuong et al., 2008).

\section{Conclusions}

Gout is a disease more and more common in the developing countries populations. Epidemiological investigations show that the number of gout cases is increasing both of women and men. The tendency is connected with the life expectancy becoming longer and longer. Older people suffer from various civilisation diseases, being one of the risks of gout occurrence. Apart from the above fact, an improper diet and lack of physical activity are among the factors increasing the risk of the growing incidence of the disease.

A proper diet in gout treatment is of a crucial importance, as well as introducing adequate lifestyle changes such as increasing the level of physical activity. In order to increase the therapeutic effect of the diet, it is worth to introduce plant-origin natural products showing the efficacy in supporting gout curing. Therapeutic activity compo- 
Jurczak, R., Reguła, J. (2016). Plant materials used in supporting the treatment of gout. Nauka Przyr. Technol., 10, 4, \#58. DOI: http://dx.doi.org/10.17306/J.NPT.2016.4.58

nents are present in plant raw materials such as: cherries, Aegopodium podagraria L., Harpagophytum procumbens (Devil's Claw), willow bark, birch leaves, and mud meadowsweet flower.

\section{References}

Al-Harbi, N. O., Al-Ashban, R. M., Shah Arif, H. (2013). Toxicity studies on Harpagophytum procumbens (Devil's claw) capsules in mice. J. Med. Plants Res., 7, 3089-3097. DOI: 10.5897/JMPR11.098

Andersen, M. L., Santos, E. H., Seabra Mde, L., da Silva, A. A., Tufik, S. (2004). Evaluation of acute and chronic treatments with Harpagophytum procumbens on Freund's adjuvant-induced arthritis in rats. J. Ethnopharmacol., 91, 325-330. DOI: 10.1016/j.jep.2004.01.003

Blando, F., Gerardi, C., Nicoletti, I. (2004). Sour Cherry (Prunus cerasus L.) Anthocyanins as Ingredients for Functional Foods. J. Biomed. Biotechnol., 5, 253-258. DOI: $10.1155 / \mathrm{S} 1110724304404136$

Błecha, K., Wawer, I. (eds.). (2011). Profilaktyka zdrowotna i fitoterapia. Rola suplementów diety, dietetycznych środków spożywczych specjalnego przeznaczenia medycznego, żywności funkcjonalnej oraz leków roślinnych. Żywiec: Wyd. Bonimed.

Christensen, L. P., Brandt, K. (2006). Bioactive polyacetylenes in food plants of the Apiaceae family: Occurrence, bioactivity and analysis. J. Pharm. Biomed. Anal., 41, 683-693. DOI: 10.1016/j.jpba.2006.01.057

Cisowski, W. (2010). Skrzyp polny - roślina lecznicza. Panacea, 4, 33, 5-7.

Denner, S. S. (2007). A review of the efficacy and safety of devil's claw for pain associated with degenerative musculoskeletal diseases, rheumatoid, and osteoarthritis. Holist Nurs. Pract., 21, 203-207. DOI: 10.1097/01.HNP.0000280932.65581.72

ESCOP Monographs (Second Edition) Supplement (2009). The Scientific Foundation for Herbal Medicinal Products, ESCOP and THIEME, 138.

ESCOP Monographs (Second Edition) (2003). The Scientific Foundation for herbal Medicinal Products, ESCOP and THIEME, 49.

Gibson, T. (2004). Clinical features of gout. In: Practical rheumatology (pp. 521-529). Ed. M. Hochberg. London: Mosby.

Gińdzieńska-Sieśkiewicz, E., Sierakowski, S., Domysławska, I., Sulik, A. (2010). Dna moczanowa - aktualne spojrzenie na diagnostykę i leczenie. Reumatologia, 48, 6, 425-428.

Grys, A. (2009). Dzika róża (Rosa canina L.) - chemizm i zastosowanie w lecznictwie. Dog rose (Rosa canina) - chemical content and medical application. Post. Fitoter., 4, 245-247.

Guasch-Ferré, M., Bulló, M., Babio, N., Martínez-González, M. A., Estruch, R., Covas, M. I., Wärnberg, J., Arós, F., Lapetra, J., Serra-Majem, L., Basora, J., Salas-Salvadó, J. (2013). Mediterranean diet and risk of hyperuricemia in elderly participants at high cardiovascular risk. J. Gerontol. A-Biol. Sci. Med. Sci., 68, 10, 1263-1270. DOI: 10.1093/gerona/glt028

Hainer, B. L., Matheson, E., Wilkes, R. T. (2014). Diagnosis, treatment, and prevention of gout. Am. Fam. Phys., 15, 90(12), 831-836.

Jacob, R. A., Spinozzi, G. M., Simon, V. A., Kelley, D. S., Prior, R. L., Hess-Pierce, B., Kader, A. A. (2003). Consumption of cherries lowers plasma urate in healthy women. J. Nutr., 133, $6,1826-1829$.

Jambor, J. (2012). Fitoterapia chorób układu moczowego. Prz. Urol., 1, 71.

Kahkonen, M. P., Hopia, A. I., Vuorela, H. J., Rauha, J. P., Pihlaja, K., Kujala, T. S., Heinonen, M. (1999). Antioxidant activity of plant extracts containing phenolic compounds. J. Agric. Food Chem., 47, 10, 3954-3962. 
Jurczak, R., Reguła, J. (2016). Plant materials used in supporting the treatment of gout. Nauka Przyr. Technol., 10, 4, \#58. DOI: http://dx.doi.org/10.17306/J.NPT.2016.4.58

Kolasinski, S. L. (2014). Food, drink, and herbs: alternative therapies and gout. Curr. Rheumatol. Rep., 16, 4, 409. DOI: 10.1007/s11926-014-0409-8

Kunstman, P., Wojcińska, M., Popławska, P. (2012). Podagrycznik pospolity (Aegopodium podagr aria L.). Post. Fitoter., 4, 244-249.

Kuo, C. F., Grainge, M. J., Zhang, W., Doherty, M. (2015). Global epidemiology of gout: prevalence, incidence and risk factors. Nat. Rev. Rheum., 11, 11, 649-662. DOI: 10.1038/nrrheum.2015.91

Lamer-Zarawska, E., Kowal-Gierczak, B., Niedworok, J. (eds.). (2012). Fitoterapia i leki roślinne. Warszawa: PZWL.

Le MyPhuong, T., Shafiu, M., Mu, W., Johnson, R. J. (2008). SLC2A9 - a fructose transporter identified as a novel uric acid transporter. Nephrol. Dial. Transpl., 23, 2746-2749. DOI: $10.1093 / \mathrm{ndt} / \mathrm{gfn} 349$

Liapina, L. A., Koval'chuk, G. A. (1993). A comparative study of the action on the hemostatic system of extracts from the flowers and seeds of the meadowsweet (Filipendula ulmaria (L.) Maxim.). Izv. Akad. Nauk. Ser. Biol., 4, 625-628.

Lis, K. (2010). Diabelska moc czarciego pazura. Reumatologia, 48, 2, 128-132.

Mahomed, I. M., Ojewole, J. A. (2004). Analgesic, antiinflammatory and antidiabetic properties of Harpagophytum procumbens DC (Pedaliaceae) secondary root aqueous extract. Phytother. Res., 18, 12, 982-989. DOI: 10.1002/ptr.1593

Majdan, M. (2013). Dna moczanowa - nowe możliwości diagnostyczne i terapeutyczne. Reumatologia, 51, 1-8. DOI: 10.5114/reum.2013.33386

Majdan, M., Borys, O. (2010). Dna i schorzenia towarzyszące podwyższonemu stężeniu kwasu moczowego. Ann. Acad. Med. Stetin., 56, Suplement 1, 34-39.

Matławska, I. (ed.). (2006). Farmakognozja. Poznań: Wyd. Akademia Medyczna im. Karola Marcinkowskiego w Poznaniu.

McCune, L. M., Kubota, C., Stendell-Hollis, N. R., Thomson, C. A. (2011). Cherries and health: a review. Crit. Rev. Food Sci. Nutr., 51, 1, 1-12. DOI: 10.1080/10408390903001719

Michalska, I., Pham, M. (2002). Kora wierzby - badania, surowiec, zastosowanie. Panacea, 1, 9-10.

Mills, S. Y., Jacoby, R. K., Chacksfield, M., Willoughby, M. (1996). Effect of a proprietary herbal medicine on the relief of chronic arthritis pain: a double-blind study. Br. J. Rheumatol., 35, 9, 874-878.

Nartowska, J. (2011). Brzoza - drzewo święte Stoi... nad całą leśną gromadą wzniesiona wysmukłością kibici i barwy powabem: Brzoza biała..., Panacea, 4, 37, 5-7.

Pieszak, M., Mikołajczak, P. Ł. (2010). Właściwości lecznicze pokrzywy zwyczajnej (Urtica dioica L.). Post. Fitoter., 4, 199-204.

Priori, N. M., Lundgaard, N. H., Light, M. E., Stafford, G. I., van Staden, J., Jäger, A. K. (2007). The polyacetylene falcarindiol with COX-1 activity isolated from Aegopodium podagraria L. J. Ethnopharmacol., 113, 176-178. DOI: 10.1016/j.jep.2007.05.005

Raciborski, F., Maślińska, M., Kłak, A., Filipiak, K. J., Szymański, F. M., Szczypior, A. (2015). Występowanie i leczenie dny moczanowej w Polsce. Analiza - wskazania - rekomendacje. Warszawa: Instytut Ochrony Zdrowia w Polsce.

Sarwa, A. (2001). Wielki leksykon roślin leczniczych. Warszawa: KiW.

Shilova, I. V., Zhavoronok, T. V., Suslov, N. I., Krasnov, E. A., Novozheeva, T. P., Veremeev, A. V., Nagaev, M. G., Petina, G. V. (2006a). Hepatoprotective and antioxidant activity of meadowsweet extract during experimental toxic hepatitis. Bull. Exp. Biol. Med., 142, 2, 216-218. DOI: $10.1007 / \mathrm{s} 10517-006-0331-9$

Shilova, I. V., Krasnov, E. A., Korotkova, E. I., Nagaev, M. G., Lukina, A. N. (2006b). Antioxidant properties of extracts from the above-ground parts of Filipendula ulmaria. Pharm. Chem. J., 40, 660-662. DOI: 10.1007/s11094-006-0214-4

Singh, J. A. (2013). Racial and gender disparities in patients with gout. Curr. Rheumatol. Rep., 15, 2, 307. DOI: 10.1007/s11926-012-0307-x 
Jurczak, R., Reguła, J. (2016). Plant materials used in supporting the treatment of gout. Nauka Przyr. Technol., 10, 4, \#58. DOI: http://dx.doi.org/10.17306/J.NPT.2016.4.58

Smolarz, H. D., Sokołowska-Woźniak, A. (2001). Aktywność farmakologiczna wyciągów z Filipendula ulmaria i Filipendula hexapetala. Post. Fitoter., 4, 12-15.

Souci, S. W., Fachmann, W., Kraut, H. (2008). Food Composition and Nutrition Tables. Medpharm Gmbh Scientific Publisher.

Stefanovic, O., Comic, L., Stanojevic, D., Solujic-Sukdolak, S. (2009). Antibacterial activity of Aegopodium podagraria L. Extracts and interaction between extracts and antibiotics. Turk. J. Biol., 33, 145-150.

Struczyński, J. (2004). Reumatoidalne zapalenie stawów (RZS). Panacea, 4, 9, 7-9.

Trąba, C., Rogut, K., Wolański, P. (2012). Rośliny dziko występujące i ich zastosowanie przewodnik po wybranych gatunkach. Rzeszów: Procarpathia.

Wang, H., Nair, M. G., Strasburg, G. M., Chang, Y. C., Booren, A. M., Gray, J. I., DeWitt, D. L. (1999). Antioxidant and antiinflammatory activities of anthocyanins and their aglycon, cyanidin, from tart cherries. J. Nat. Prod., 62, 2, 294-296. DOI: 10.1021/np980501m

Wolski, T., Baj, T., Ludwiczuk, A., Głowniak, K., Niedźwiecki, R. (2010). Hakorośl rozesłana (Harpagophytum procumbens DC) - roślinny surowiec o wielokierunkowym działaniu farmakologicznym. Post. Fitoter., 1, 13-22.

Zimmermann-Górska, I. (2006). Dna moczanowa. Reumatologia, 44, 61-62.

Zhang, W., Doherty, M., Pascual, E., Bardin, T., Barskova, V., Conaghan, P., Gerster J., Jacobs, J., Leeb, B., Lioté, F., McCarthy, G., Netter, P., Nuki, G., Perez-Ruiz, F., Pignone, A., Pimentão, J., Punzi, L., Roddy, E., Uhlig, T., Zimmermann-Górska, I. (2006). Eular evidence based recommendations for gout. Part I: Diagnosis. Report of a task force of the standing committee for international clinical studies including therapeutics (ESCISIT). Ann. Rheum. Dis., 65, 13011311. DOI: 10.1136/ard.2006.055269

Zhang, Y., Neogi, T., Chen, C., Chaisson, C., Hunter, D. J., Choi, H. K. (2012). Cherry consumption and decreased risk of recurrent gout attacks. Arthritis Rheum., 64, 12, 4004-4011. DOI: $10.1002 /$ art.34677

\title{
SUROWCE ROŚLINNE WYKORZYSTYWANE WE WSPOMAGANIU LECZENIA DNY MOCZANOWEJ
}

\begin{abstract}
Streszczenie
Dna moczanowa (artretyzm, skaza moczanowa) jest zaliczana do chorób reumatycznych. W wyniku zaburzeń metabolizmu kwasu moczowego jego kryształki odkładają się w tkankach i narządach. Powoduje to dolegliwości bólowe stawów i wytwarzanie tzw. guzków dnawych i nerki dnawej. Wartość graniczna kwasu moczowego we krwi to $6,5 \mathrm{mg} \%$. Na całym świecie, szczególnie w krajach wysoko rozwiniętych, odnotowuje się coraz więcej zachorowań na dnę moczanową. Częstość zachorowań jest większa u mężczyzn niż u kobiet, wśród których wzrasta dopiero w okresie pomenopauzalnym. Metody leczenia bazują na kompleksowym podejściu do pacjentaprzede wszystkim na zmianie stylu życia (wprowadzenie specjalistycznej diety i zwiększenie aktywności fizycznej) oraz na wprowadzaniu leków przeciwbólowych i przeciwzapalnych, które usuwają także nadmiar kwasu moczowego. Istotne znaczenie w leczeniu dny moczanowej mają także surowce roślinne o sile działania porównywalnej do siły działania leków, ale powodujące mniejsze efekty uboczne. We wspomaganiu leczenia dny moczanowej należy zwrócić uwagę na takie rośliny jak: podagrycznik pospolity, korzeń hakorośli, kora wierzby, liście brzozy, kwiat wiązówki błotnej, wiśnie, ale także nawłoć pospolita, pokrzywa, skrzyp czy dzika róża.
\end{abstract}

Słowa kluczowe: artretyzm, dieta, rośliny, puryny, substancje bioaktywne 
Jurczak, R., Reguła, J. (2016). Plant materials used in supporting the treatment of gout. Nauka Przyr. Technol., 10, 4, \#58. DOI: http://dx.doi.org/10.17306/J.NPT.2016.4.58

Corresponding address - Adres do korespondencji:

Julita Reguła, Katedra Higieny Żywienia Człowieka, Uniwersytet Przyrodniczy w Poznaniu, ul. Wojska Polskiego 31/33,60-624 Poznań, Poland, e-mail: jumar@up.poznan.pl

Accepted for publication - Zaakceptowano do opublikowania:

19.12.2016

For citation - Do cytowania:

Jurczak, R., Reguta, J. (2016). Plant materials used in supporting the treatment of gout. Nauka Przyr. Technol., 10, 4, \#58. DOI: http://dx.doi.org/10.17306/J.NPT.2016.4.58 